\title{
Rewiring of the jasmonate signaling pathway in Arabidopsis during insect herbivory
}

\section{Adriaan Verhage ${ }^{1}$, Ido Vlaardingerbroek ${ }^{1}$, Ciska Raaymakers ${ }^{2}$, Nicole M. Van Dam ${ }^{2,3}$, Marcel Dicke $^{4}$, Saskia C. M. Van Wees ${ }^{1}$ and Corné M. J. Pieterse ${ }^{1,5}{ }^{*}$}

1 Plant-Microbe Interactions, Department of Biology, Faculty of Science, Utrecht University, Utrecht, Netherlands

${ }^{2}$ Multitrophic Interactions, Netherlands Institute of Ecology, Wageningen, Netherlands

${ }^{3}$ Ecogenomics, Institute for Water and Wetland Research, Radboud University Nijmegen, Nijmegen, Netherlands

${ }^{4}$ Laboratory of Entomology, Wageningen University, Wageningen, Netherlands

${ }^{5}$ Centre for BioSystems Genomics, Wageningen, Netherlands

\section{Edited by:}

Adi Avni, Tel Aviv University, Israel

Reviewed by:

Douglas R. Cook, University of California-Davis, USA

Claus Wasternack, Leibniz Institute of

Plant Biochemistry, Germany

${ }^{*}$ Correspondence:

Corné M. J. Pieterse, Plant-Microbe

Interactions, Department of Biology,

Faculty of Science, Utrecht

University, P.O. Box 800.56, 3508 TB

Utrecht, Netherlands.

e-mail:c.m.j.pieterse@uu.nl
Plant defenses against insect herbivores and necrotrophic pathogens are differentially regulated by different branches of the jasmonic acid (JA) signaling pathway. In Arabidopsis, the basic helix-loop-helix leucine zipper transcription factor (TF) MYC2 and the APETALA2/ETHYLENE RESPONSE FACTOR (AP2/ERF) domain TF ORA59 antagonistically control these distinct branches of the JA pathway. Feeding by larvae of the specialist insect herbivore Pieris rapae activated MYC2 transcription and stimulated expression of the MYC2-branch marker gene VSP2, while it suppressed transcription of ORA59 and the ERF-branch marker gene PDF1.2. Mutant jin 1 and jar1-1 plants, which are impaired in the MYC2-branch of the JA pathway, displayed a strongly enhanced expression of both ORA59 and PDF1.2 upon herbivory, indicating that in wild-type plants the MYC2-branch is prioritized over the ERF-branch during insect feeding. Weight gain of $P$. rapae larvae in a no-choice setup was not significantly affected, but in a two-choice setup the larvae consistently preferred jin1 and jar1-1 plants, in which the ERF-branch was activated, over wild-type Col-0 plants, in which the MYC2-branch was induced. In MYC2- and ORA59-impaired jin1-1/RNAi-ORA59 plants this preference was lost, while in ORA59-overexpressing 35S:ORA59 plants it was gained, suggesting that the herbivores were stimulated to feed from plants that expressed the ERF-branch rather than that they were deterred by plants that expressed the MYC2branch. The feeding preference of the $P$. rapae larvae could not be linked to changes in glucosinolate levels. Interestingly, application of larval oral secretion into wounded leaf tissue stimulated the ERF-branch of the JA pathway, suggesting that compounds in the oral secretion have the potential to manipulate the plant response toward the caterpillarpreferred ERF-regulated branch of the JA response. Our results suggest that by activating the MYC2-branch of the JA pathway, plants prevent stimulation of the ERF-branch by the herbivore, thereby becoming less attractive to the attacker.

Keywords: plant defense signaling, jasmonic acid, plant-insect interactions, hormone crosstalk, Pieris rapae

\section{INTRODUCTION}

Plants possess a powerful innate immune system by which they recognize non-self molecules or signals from injured cells, and respond by activating an effective defense response (Jones and Dangl, 2006; Howe and Jander, 2008). Defense mechanisms that are induced upon attack by herbivorous insects involve direct defenses such as the production of proteinase inhibitors and glucosinolates that target essential physiological processes in the insect, and indirect defenses such as the emission of volatiles that attract parasitoids and predators of the herbivores that feed on the plant (Howe and Jander, 2008; Dicke et al., 2009; Hopkins et al., 2009). The plant hormone jasmonic acid (JA) and its oxylipin derivatives (collectively called here jasmonates, JAs) are key players in the regulation of induced plant responses against herbivory (Koo and Howe, 2009). JAs also play important roles in plant defense against necrotrophic pathogens (Glazebrook, 2005; Laluk and Mengiste, 2010). However, the JAs-controlled responses to necrotrophs and insect herbivores seem to be regulated via different branches of the JA signaling pathway (Lorenzo and Solano, 2005; Kazan and Manners, 2008).

Upon pathogen or insect attack, JAs are rapidly synthesized via the oxylipin biosynthesis pathway (Wasternack, 2007; Gfeller et al., 2010). Through the JA conjugate synthase JAR1, JA can be readily conjugated to amino acids such as isoleucine (Staswick and Tiryaki, 2004), resulting in biologically highly active specific enantiomer of jasmonoyl-isoleucine (JA-Ile; Fonseca et al., 2009). The F-box protein CORONATINE INSENSITIVE 1 (COI1) functions as a JA-Ile receptor in the E3 ubiquitin-ligase Skip-CullinF-box complex SCFCOI1 (Yan et al., 2009; Sheard et al., 2010). Binding of JA-Ile to COI1 leads to degradation of JASMONATE ZIM-domain (JAZ) transcriptional repressor proteins via the proteasome (Chini et al., 2007; Thines et al., 2007). Consequently, the 
physical interaction of JAZ proteins with transcriptional activators, leading to repression of JA signaling in resting cells, is broken in JA-stimulated cells, which results in the activation of a large number of JA-responsive genes (Chini et al., 2007; Memelink, 2009).

Transcriptional changes in response to diverse JA-inducing pathogens and insect herbivores show limited overlap, suggesting that the context in which the JA signal is perceived is crucial in tuning the JA response (De Vos et al., 2005; Pauwels et al., 2009). The plant hormones ethylene (ET), abscisic acid (ABA), and salicylic acid (SA), which in various combinations and concentrations can be part of the signal signature that is produced upon pathogen or insect attack, emerged as important differential regulators of the JA response (Leon-Reyes et al., 2009, 2010; Pieterse et al., 2009). While SA generally acts antagonistically on the JA response (Koornneef et al., 2008; Verhage et al., 2010), ET and ABA each co-regulate a different branch of the JA response (Adie et al., 2007; Kazan and Manners, 2008).

JA and ET are often simultaneously produced upon infection by necrotrophic pathogens (De Vos et al., 2005). In Arabidopsis thaliana (Arabidopsis), JA, and ET signaling act synergistically on the expression of defense-related genes, such as PLANT DEFENSIN1.2 (PDF1.2; Penninckx et al., 1998). Two members of the APETALA2/ETHYLENE RESPONSE FACTOR (AP2/ERF) family of transcription factors (TFs), ERF1 and ORA59, emerged as principal integrators of JA and ET signaling (Lorenzo et al., 2003; Pré et al., 2008). Both TFs activate the ERF-branch of the JA pathway and confer resistance to necrotrophic pathogens (Berrocal-Lobo et al., 2002; Pré et al., 2008). RNAi-ORA59 plants were demonstrated to be completely blocked in JA/ET-induced PDF1.2 expression, indicating that ORA59 is required for this response. Another important master regulator of JA-responsive gene expression is the basic helix-loop-helix leucine zipper TF MYC2 (originally called JIN1 for JASMONATE INSENSITIVE1). On the one hand, MYC2 acts in concert with ABA signaling in negatively regulating the ERF-branch of the JA pathway (Anderson et al., 2004; Lorenzo et al., 2004). On the other hand, MYC2 functions as a transcriptional activator of genes in the MYC2-branch of the JA pathway, including the JA marker gene VEGETATIVE STORAGE PROTEIN2 (VSP2; Dombrecht et al., 2007). The MYC2branch of the JA pathway is associated with the wound-response and is thought to contribute to defense against insect herbivores (Lorenzo et al., 2004; Dombrecht et al., 2007; Fernandez-Calvo et al., 2010), although it has also been demonstrated to play a role in priming for enhanced pathogen defense (Pozo et al., 2008; Van der Ent et al., 2009).

Continuous co-evolution between plants and herbivores has provided the latter with mechanisms to avoid, suppress, or eliminate host defenses (Schoonhoven et al., 2005; Pieterse and Dicke, 2007; Kant et al., 2008; Walling, 2008). Larvae of the specialist insect herbivore Pieris rapae (small cabbage white butterfly) feed exclusively on crucifers. In the field, they have been reported to feed on many brassicaceous species, including Arabidopsis (Yano and Ohsaki, 1993). P. rapae larvae are well adapted to the induced defenses of brassicaceous species. For instance, $P$. rapae produces an enzyme that redirects the myrosinase-driven conversion of glucosinolates from the toxic isothiocyanates toward the less noxious nitriles. By detoxifying the herbivory-triggered "mustard oil bomb," $P$. rapae caterpillars efficiently avoid exposure to these highly toxic chemicals (Wittstock et al., 2004). However, Arabidopsis still possesses additional direct defenses that are active against this specialist herbivore as demonstrated by elevated resistance in systemic undamaged plant parts induced by prior feeding by $P$. rapae (De Vos et al., 2006). Herbivory by $P$. rapae leads to increased production of JAs and extensive reprogramming of the expression of JA-responsive genes, many of which are associated with plant defense (Reymond et al., 2000, 2004; De Vos et al., 2005). Transcriptional changes inflicted by mechanical wounding or insect feeding overlap only marginally (Reymond et al., 2000), suggesting that insect-derived cues play an important role in the modulation of the plant's transcriptional response to herbivory.

Ever since the seminal paper by Ehrlich and Raven (1964), ecologists have intensely debated the arms race between plants and herbivorous insects (Thompson, 1994; Schoonhoven et al., 2005). However, knowledge of the underlying mechanisms is still relatively limited in comparison to the well-studied mechanisms involved in the arms race between pathogens and their host plants (Jones and Dangl, 2006). Induced plant responses to insect herbivory consist of a mix of direct and indirect plant defenses aimed at limiting insect performance, and of insect-mediated plant responses that favor the insect herbivore. Here, we investigated the response of Arabidopsis to herbivory by the specialist $P$. rapae. We demonstrate that oral secretions of $P$. rapae induce the ERF-branch of the JA pathway, whereas feeding larvae activate the antagonistic counterpart of the JA response that is regulated by MYC2. We establish that activation of the MYC2-branch is favorable for the plant, not because it would actively deter caterpillars that feed on the plant, but because it suppresses the ERF-branch of the JA pathway for which the caterpillars have a preference. This "hidethe-candy" strategy of the plant sheds new light on the mechanisms involved in the evolutionary arms race between plants and their herbivorous enemies.

\section{RESULTS \\ HERBIVORY ACTIVATES THE MYC2-BRANCH AND SUPPRESSES THE ERF-BRANCH OF THE JA PATHWAY}

The transcriptional response of Arabidopsis to P. rapae feeding is predominantly regulated via the JA signaling pathway (Reymond et al., 2004; De Vos et al., 2005). To investigate the significance of the MYC2- and the ERF-branch of the JA pathway in this response, we monitored the expression of the marker genes PDF1.2 (ERFbranch) and VSP2 (MYC2-branch). Caterpillars of larval stage L1 were allowed to feed on leaves of Arabidopsis wild-type Col-0 plants for $24 \mathrm{~h}$, after which they were removed (Figure 1A). Damaged leaves of $P$. rapae-infested plants were harvested for gene expression analysis. Caterpillar feeding activated VSP2 in infested tissue (from $6 \mathrm{~h}$ onward; Figure 1B), which correlated with the induced expression pattern of MYC2. PDF1.2 was only mildly induced in herbivore-damaged leaves (24 h onward). After $12 \mathrm{~h}$ of feeding, VSP2 and MYC2 showed a marked decrease in expression level. Since this time point is harvested in the dark period, it confirms previous findings that JA signaling and responses to herbivory are attenuated during shading conditions (Moreno et al., 2009). 
A

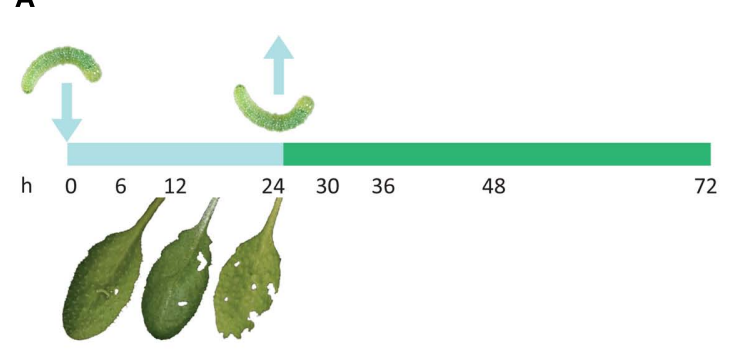

B

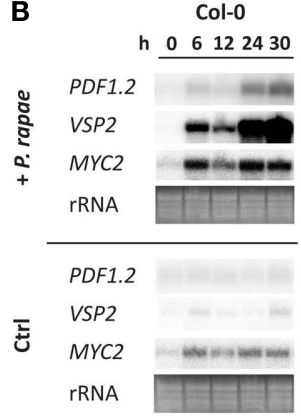

C

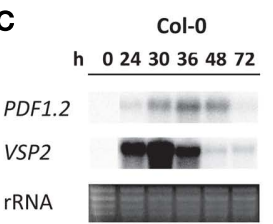

jin1-1

jin1-2

jin1-7

jin1-10

jar1-1
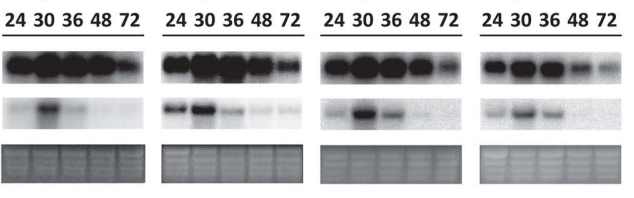

2430364872

D

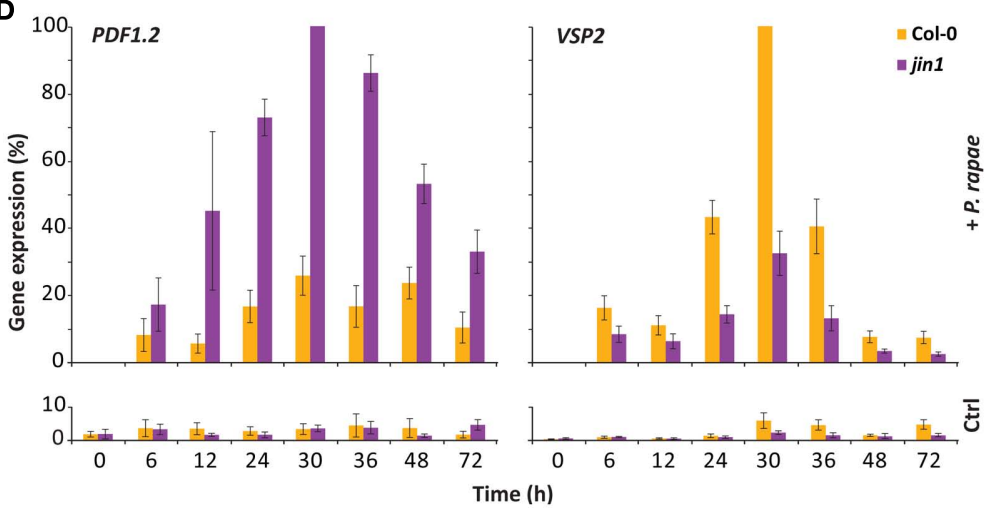

FIGURE 1 | Effect of MYC2 on the differential JA response during herbivory by $\boldsymbol{P}$ rapae. (A) Experimental setup for the gene expression analyses of the Arabidopsis- $P$. rapae interaction. First-instar larvae of $P$. rapae were allowed to feed on 6-week-old Arabidopsis plants, after which they were removed. The time scale shows the harvest time points that were used in the different experiments. (B) Northern blot analysis of JA-responsive PDF1.2 (marker for ERF-branch), VSP2 (marker for MYC2-branch), and MYC2 gene expression in leaves of non-treated control
(Ctrl) and $P$. rapae-infested wild-type Col-0 plants. (C) Northern blot analysis of PDF1.2 and VSP2 transcription in Col-0, and mutant jin 1 and jar1-1 plants. Time point $\mathrm{O}$ h is only given for Col-0 but was similar in the other genotypes (see also Figure 1D). To check for equal loading, rRNA bands were stained with ethidium bromide. (D) Signal intensities of PDF1.2 and VSP2 mRNA on the northern blots of multiple experiments were quantified using a phosphor imager and plotted relative to the highest level (set at 100\%). Error bars represent \pm SE.
To investigate whether herbivory-mediated activation of the MYC2-branch of the JA pathway was associated with a concomitant suppression of the ERF-branch, we monitored the expression of PDF1.2 and VSP2 in jin1 mutants that are impaired in MYC2 function (Anderson et al., 2004; Lorenzo et al., 2004). In Col0 plants, $24 \mathrm{~h}$ of $P$. rapae feeding resulted in the accumulation of high levels of VSP2 mRNA, which slowly declined back to basal levels $24 \mathrm{~h}$ after removal of the caterpillars (Figure 1C). In MYC2-defective jin1-1, jin1-2, jin1-7, and jin1-10 mutants, the level of VSP2 mRNA was strongly reduced, confirming that MYC2 is an important regulator of VSP2 transcription. Interestingly, the jin 1 mutants accumulated high levels of PDF1.2 mRNA after $24 \mathrm{~h}$ of herbivory, which slowly declined after removal of the $P$. rapae larvae. Figure $1 \mathrm{D}$ displays the average relative expression levels of PDF1.2 and VSP2 in Col-0 and jin 1 mutants during $24 \mathrm{~h}$ of insect feeding $(0-24 \mathrm{~h})$ and up to 3 days after removal of the herbivores $(24-72 \mathrm{~h})$. Together, these results indicate that in wild-type plants feeding by $P$. rapae activates the expression of VSP2 while it suppresses PDF1.2 transcription, and that in MYC2impaired jin1 mutants these expression patterns are swopped. Mutant jar1-1 plants, which are impaired in the production of bioactive JA-Ile (Staswick and Tiryaki, 2004) and as a result display reduced MYC2-dependent transcriptional activity, showed a similar PDF1.2 expression pattern as the jin1 mutants (Figure 1C), corroborating the role of MYC2 in antagonizing the ERF-branch of the JA pathway during herbivory.

\section{ROLE OF ORA59 IN HERBIVORE-MEDIATED SUPPRESSION OF THE ERF-BRANCH OF THE JA PATHWAY}

Since the AP2/ERF-type TF ORA59 is a major regulator of the ERF-branch of the JA pathway (Pré et al., 2008), we monitored the expression of ORA59 in P. rapae-infested Col-0 and jin1-2 plants. 
Figure 2A shows that herbivore-induced ORA59 expression was significantly higher in jin1-2 than in Col-0 plants. To investigate the role of ORA59 in the activation of the ERF-branch of the JA pathway during $P$. rapae feeding, we analyzed herbivore-induced transcription of PDF1.2, VSP2, ORA59, and MYC2 in ORA59silenced RNAi-ORA59 plants (Pré et al., 2008). As expected, ORA59 and PDF1.2 transcript levels were virtually undetectable in herbivore-infested RNAi-ORA59 plants (Figure 2B). Conversely, MYC2 levels were enhanced in RNAi-ORA59 plants in comparison to Col-0, suggesting that in wild-type plants ORA59 attenuates the MYC2-branch of the JA pathway during insect herbivory. In jin1-1 plants, ORA59 and PDF1.2 mRNAs accumulated to high levels after $P$. rapae feeding, again indicating that the MYC2mediated suppression of the ERF-branch was alleviated in this mutant. Silencing of ORA59 in the jin1-1 mutant background
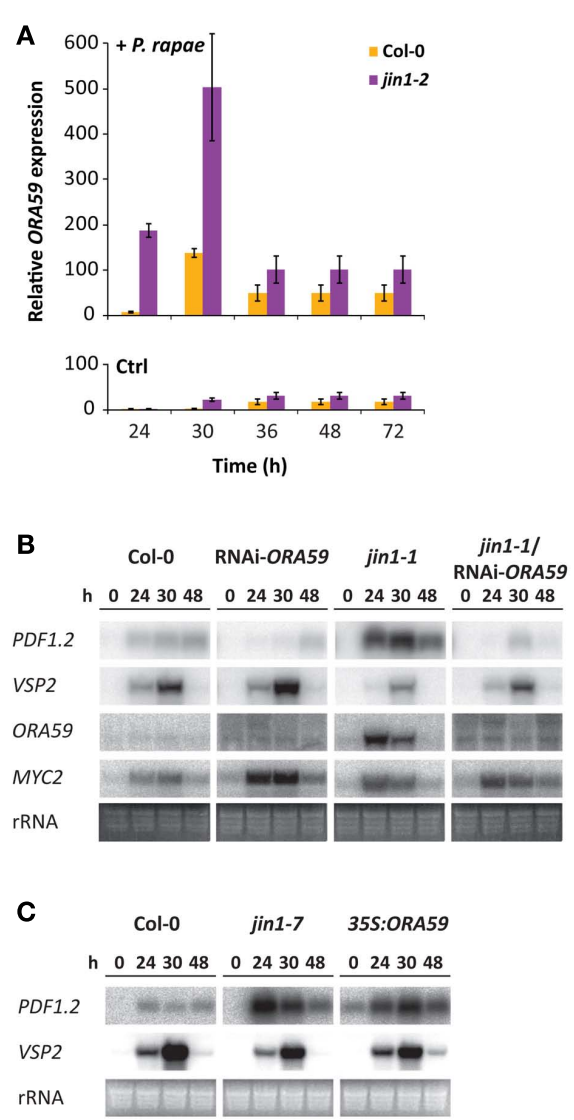

FIGURE 2 | Effect of ORA59 on the differential JA response during $\boldsymbol{P}$ rapae feeding. (A) RT-qPCR analysis of ORA59 transcript levels (relative to At1g13320) in non-treated and P. rapae-infested Arabidopsis Col-0 and jin 1-2 plants at $24,30,36,48$, and $72 \mathrm{~h}$ after removal of $P$. rapae caterpillars that had fed on the tissue for $24 \mathrm{~h}$. Transcript levels relative to non-treated Col-0 at $t=24 \mathrm{~h}$, which was set at 1 , are plotted. (B) Northern blot analysis of PDF1.2, VSP2, ORA59, and MYC2 transcript levels in Col-0, RNAi-ORA59, jin 1-1 and jin 1-1/RNAi-ORA59 plants at different time points after $P$. rapae feeding. (C) Northern blot analysis of PDF1.2 and VSP2 transcript levels in Col-0, jin 1-7 and 35S:ORA59 plants at different time points after $P$. rapae feeding. To check for equal loading, rRNA bands were stained with ethidium bromide. The experiments were repeated with similar results.
(jin1-1/RNAi-ORA59) strongly suppressed the $P$. rapae-induced expression levels of ORA59 and PDF1.2 that were apparent in the jin1-1 single mutant. Together, these results point to a model in which the suppression of the ERF-branch of the JA pathway by MYC2 during insect herbivory acts through an antagonistic effect of MYC2 on ORA59 transcription. To verify this hypothesis we analyzed the expression of PDF1.2 and VSP2 in P. rapae-infested 35S:ORA59 plants in which any suppressive effect on the ORA59 promoter would be bypassed by the $35 S$-driven overexpression of ORA59. Figure 2C shows that $P$. rapae feeding induced the expression of PDF1.2 in 35S:ORA59 plants to the same level as that observed in jin1-7 plants, suggesting that in wild-type plants repression of ORA59 is a limiting factor for the herbivore-induced expression of the ERF-branch. These results support the notion that MYC2-mediated suppression of the ERF-branch of the JA pathway during $P$. rapae feeding is likely to be caused by suppression of ORA59 transcription, rather than by suppression of ORA59 activity.

\section{EFFECT OF THE MYC2- AND THE ERF-BRANCH OF THE JA PATHWAY ON INSECT PERFORMANCE IN A NO-CHOICE ASSAY}

To investigate whether prioritization of the MYC2-branch over the ERF-branch during herbivory affects insect performance, we assessed growth of $P$. rapae larvae over a 10-day feeding period. Feeding of $P$. rapae L1 instar larvae on the JA receptor mutant coil-1( $g l 1)$ [in glabrous Col-5( $g l 1)$ background] resulted in a moderate but significant increase in weight gain of the caterpillars (Figure 3A), confirming previous findings that JA signaling is involved in resistance against grazing by $P$ rapae caterpillars (Reymond et al., 2004; Bodenhausen and Reymond, 2007; Van Oosten et al., 2008). As shown previously (Reymond et al., 2004), P. rapae larvae gained significantly more weight on glabrous Col-5(gl1) than on Col-0, which has a normal trichome phenotype (Figure 3B). However, neither the jin 1-1 $(g l 1)$ mutation in the glabrous Col-5(gl1) background, nor the jin1-7 mutation in the Col-0 background had an effect on the performance of $P$. rapae (Figures $3 \mathbf{B}, \mathbf{C}$ ). Also on mutant jarl-1 and the ORA59-silenced lines RNAi-ORA59\#4 and RNAi-ORA59\#9 (Pré et al., 2008) growth of $P$. rapae was not majorly affected (Figures 3D,E). Together, these results indicate that the COI1regulated JA-dependent defenses that negatively affect growth of the specialist herbivore $P$. rapae, are not seriously impaired in genotypes affected in either the MYC2- or ERF-branch of the JA pathway.

\section{EFFECT OF THE MYC2-BRANCH OF THE JA PATHWAY ON INSECT PREFERENCE IN A TWO-CHOICE ASSAY}

One of the ways by which plants can prevent loss of tissue during herbivory is by activating defenses that deter the attacker. We investigated the effect of the MYC2-branch of the JA pathway on the feeding preference of $P$. rapae caterpillars in a two-choice assay. To this end, four plants were placed in a two-choice arena $(n=20)$ consisting of two plants per genotype (Figure 4A). The plants in each arena were in physical contact with each other to allow the caterpillars to freely move from one plant to the other. At the start of the assay, two first-instar larvae were placed on each plant (eight larvae per arena) and after 4 days the number of caterpillars 


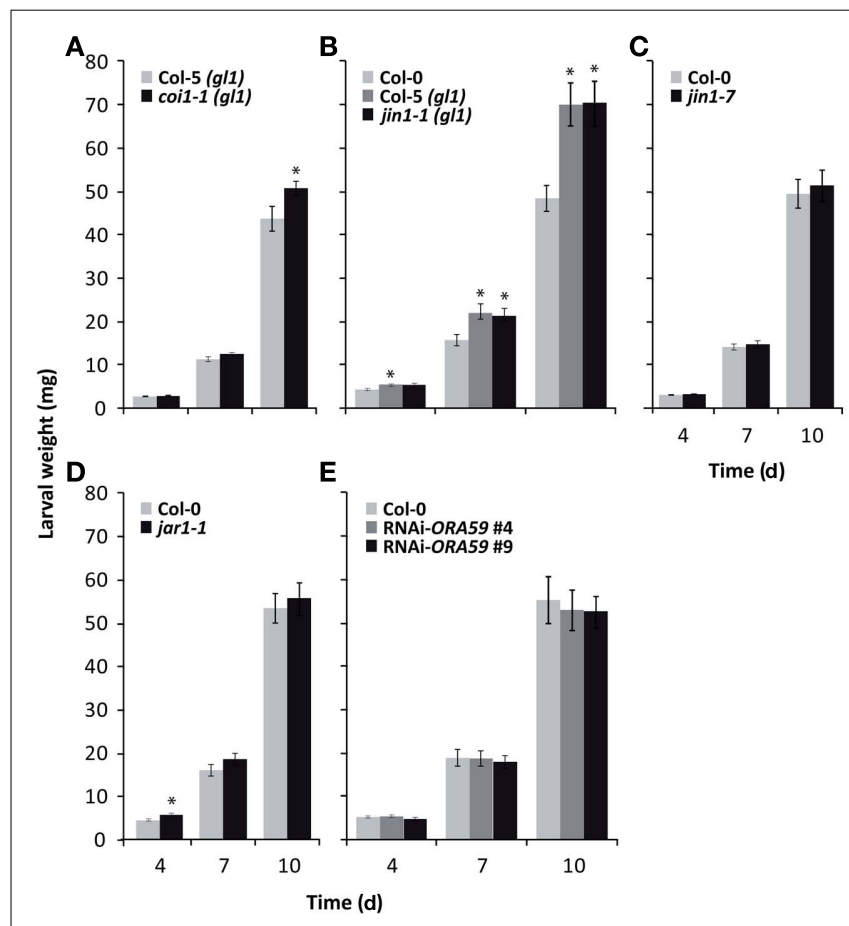

FIGURE 3 | Growth of $P$. rapae larvae on Arabidopsis genotypes that are impaired in the JA response. Growth of first-instar larvae of $P$. rapae was assessed on coi1-1(g/1) (A), jin1-1(g/1) (B), jin 1-7 (C), jar1-1 (D), and RNAi-ORA59\#4 and \#9 (E). The genotypes were compared with their respective trichome-containing (Col-0) and trichome-less [Col-5(g/1)] wild-type backgrounds. Larval fresh weight was determined after 4, 7, and 10 days of feeding. The values represent means ( \pm SE) of \pm 45 larvae per genotype. Asterisks indicate statistically significant differences in comparison to similarly treated wild-type plants (Dunnett's pairwise multiple comparison $t$-test, $p<0.05$ )

per plant genotype was determined in 20 independent two-choice arenas. MYC2-branch-related mutants jin1-1, jin1-7, and jar1-1 accommodated significantly more $P$. rapae larvae at the end of the two-choice assay than did Col-0 plants (frequency distribution of the larvae over mutant and wild-type plants: 60:40, 60:40, and 62:38\%, respectively; Figures 4B1,B2,C2). When comparing jin17 with jar1-1 in the two-choice test, the frequency distribution of the larvae over both mutants was similar (50:50\%; Figure 4B3). These results suggest that the MYC2-branch of the JA pathway regulates defense responses that affect the feeding preference of $P$. rapae larvae, and that the jin 1 and the jarl mutations affect this MYC2-dependent trait to a similar extent.

\section{P. RAPAE LARVAE PREFER TO FEED ON PLANTS THAT EXPRESS THE ERF-BRANCH OF THE JA PATHWAY}

Because genetic obstruction of the MYC2-branch of the JA pathway alleviates the suppression of the ERF-branch, the preference of the $P$. rapae larvae for jin 1 over Col- 0 plants may be caused by (1) a deterring effect of the MYC2-branch that is activated in Col-0 plants upon herbivory, or (2) a feeding stimulatory effect of the ERF-branch that is induced in jin1 plants. To test this, we compared the following Arabidopsis genotypes, which were demonstrated to be affected in MYC2- and/or ERF-regulated gene expression (Figures 1 and 2), in the two-choice assay: Col0 (MYC2-branch on, ERF-branch off), jin1-1 (MYC2-branch off, ERF-branch on), RNAi-ORA59 (MYC2-branch on, ERF-branch off), 35S:ORA59 (MYC2-branch off, ERF-branch on), and jin11/RNAi-ORA59 (both branches off). Figure 4C3 shows that mutant jin1-1 plants accommodated significantly more caterpillars than did jin1-1/RNAi-ORA59 plants (frequency distribution 59:41\%), suggesting that $P$. rapae larvae preferred feeding from plants that expressed the ERF-branch of the JA pathway, rather than that they were deterred by plants that expressed the MYC2-branch. Col-0 and jin1-1/RNAi-ORA59 accommodated similar numbers of $P$. rapae larvae (frequency distribution 49:51\%; Figure 4C4), indicating that activation of the MYC2-branch of the JA pathway in Col-0 did not make the plants less preferred than jin1-1/RNAi-ORA59 plants in which neither of the two branches were activated. The frequency distribution of the caterpillars over RNAi-ORA59 and Col-0 plants did not significantly differ (frequency distribution 55:45\%; Figure 4C1), but the small trend toward RNAi-ORA59 supports that the larvae were not deterred by stronger activation of the MYC2-branch in RNAi-ORA59 plants (Figure 2B). ORA59-overexpressing 35S:ORA59 plants (Pré et al., 2008) accommodated significantly more caterpillars then did Col0 plants (frequency distribution 63/37\%; Figure 4C5), confirming that $P$. rapae larvae prefer feeding from plants that express the ERF-branch of the JA pathway upon herbivory.

Interestingly, the JA receptor mutant coil-1, which is fully blocked in the capacity to express either the MYC2-branch or the ERF-branch of the JA pathway, accommodated more P. rapae larvae in the two-choice assay than did wild-type Col-0 (Figure 4D1). In Col-0 the ERF-branch is suppressed by the MYC2-branch but still displays low levels of ORA59 and PDF1.2 expression upon $P$. rapae feeding. Nevertheless, $P$. rapae larvae preferred coil-1 over Col-0 plants. This suggests that besides the feeding stimulatory effect that is mediated by the ERF-branch, a COI1-dependent deterring component affects $P$. rapae performance in this system as well.

\section{ORAL SECRETION OF P. RAPAE STIMULATES THE ERF-BRANCH OF THE JA PATHWAY}

The observed attraction of $P$. rapae larvae toward plants expressing the ERF-branch of the JA pathway (Figures 4B,C), prompted us to investigate whether $P$. rapae has ways to actively stimulate this favorable branch of the JA response. To this end, we simulated caterpillar feeding by mechanically damaging the leaves with three needle pricks per leaf and applying oral secretion of $P$. rapae into the wounds. Wounding transiently activated VSP2 transcription, which peaked at $6 \mathrm{~h}$ after wounding and leveled off to undetectable levels at $24 \mathrm{~h}$ after wounding (Figure 5A), confirming previous findings that wounding activates the MYC2-branch of the JA pathway (Lorenzo et al., 2004). Mechanical damage alone did not significantly activate the ERF-branch marker PDF1.2 (Figures 5A,B). However, when oral secretion of $P$. rapae was introduced in the wounds, PDF1.2 was strongly activated at 24 and $48 \mathrm{~h}$ after the treatment (Figure 5B). These results suggest that elicitors in the oral secretion of the caterpillars have the potential to activate the ERF-branch of the JA pathway, possibly to improve the quality for consumption. 

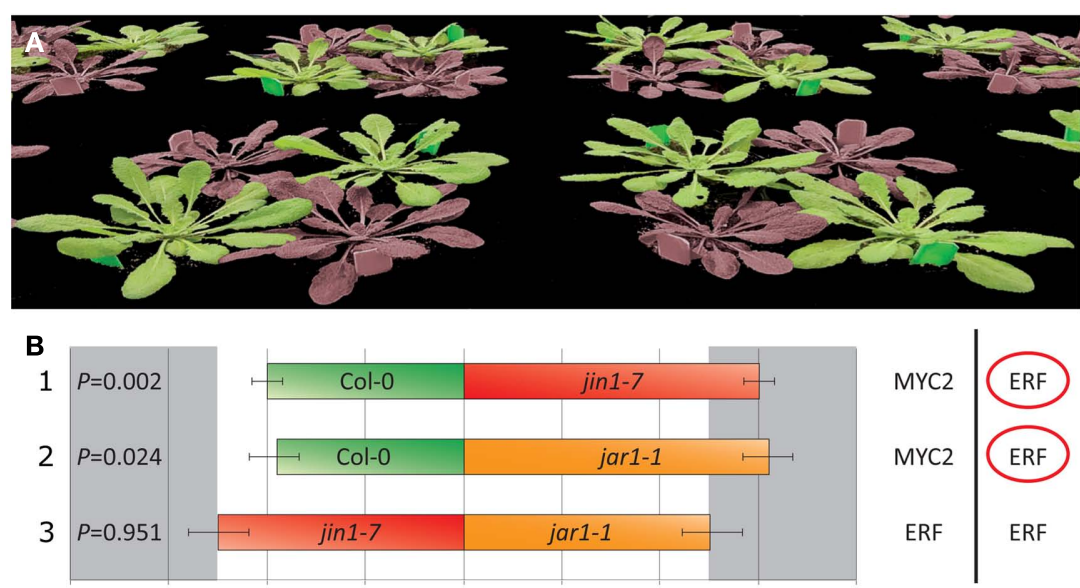

C
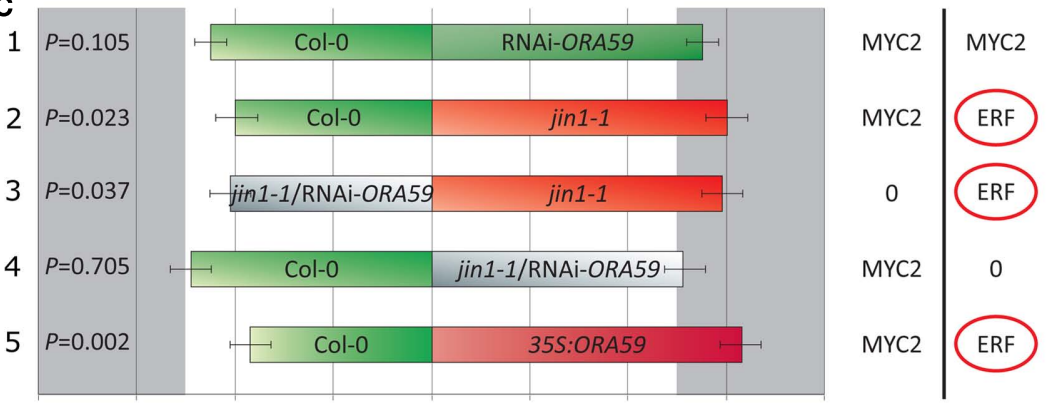

D
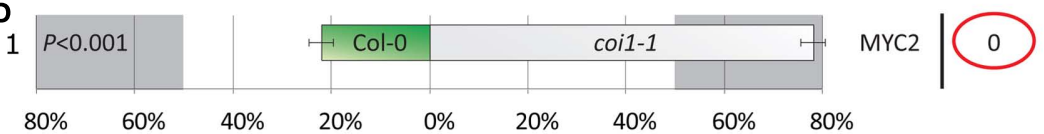

FIGURE 4 | Effect of the MYC2- and ERF-branch of the JA pathway on the preference of $\boldsymbol{P}$ rapae in two-choice tests. In the two-choice arena, two first-instar larvae of $P$. rapae were placed on each plant (A). Each two-choice arena $(n=20)$ consisted of two 6-week-old plants of each genotype (total eight larvae per arena). After 4 days the number of caterpillars on each genotype was determined. The preference of $P$. rapae larvae was tested in combinations with wild-type Col-0 plants and genotypes that are affected in the MYC2-branch of the JA pathway (B1-3) genotypes that are affected in the ERF-branch and/or the MYC2-branch of the JA pathway (C1-5), and mutant coi1-1 plants that are completely blocked in JA signaling (D1). The right panel displays which branch of the JA pathway is predominantly activated in the corresponding genotypes that are displayed in the left panel. The " 0 " indicates that neither of the branches of the JA pathway was activated. Displayed are the average percentages $( \pm S E)$ of the distribution of the $P$. rapae larvae over the two genotypes ( $x$-axis). Statistically significant differences from the $50 \%$ percentile were analyzed using a Student's $t$-test. In cases of statistically significant differences (Student's $t$-test; $p<0.05$ ), the preferred branch of the JA pathway is marked with a red circle. Experiments were repeated with similar results.

\section{P. RAPAE FEEDING PREFERENCE IS NOT AFFECTED BY PLANT GLUCOSINOLATE COMPOSITION}

Glucosinolates have been demonstrated to influence the behavior of P. rapae (Schoonhoven and Van Loon, 2002). To investigate whether the observed preference of $P$. rapae larvae for plants expressing the ERF-branch of the JA pathway is associated with a change in glucosinolate composition, first-instar $P$. rapae larvae were allowed to feed for 4 days, after which damaged plant tissue was collected and analyzed for glucosinolate content. Total glucosinolate levels were measured in both infested and noninfested Arabidopsis plants. In Col-0, coil-1, jin1-7, jar1-1, and RNAi-ORA59 plants, caterpillar feeding significantly induced the accumulation of aliphatic and indole glucosinolates (Figure 6). The constitutive levels of both the total aliphatic and indole glucosinolates, as determined in non-infested plants, were found to be similar in most plant genotypes tested (Figure 6). However, the basal levels of three out of four indole glucosinolate forms (4OH, GBC, and NEO) were reduced in coil-1 and a decrease of one of the indole glucosinolate forms $(4 \mathrm{MeOH})$ was also found for jar1-1 (Table 1). Although the P. rapae-induced levels of specific aliphatic and indole glucosinolate forms differed between Col-0 and the different genotypes tested (Table 1), no consistent pattern was observed that correlated with the preference of the $P$. rapae larvae for Arabidopsis genotypes that activated the ERF-branch of the JA pathway after herbivory (jin1-7 and jar1-1) over the ones that activated the MYC2-branch (Col-0 and RNAiORA59). For example, the levels of four aliphatic and three indole glucosinolates were significantly enhanced in jin1-7 over Col-0 (Table 1), whereas in jar1-1 the majority of these glucosinolates were not different from Col-0 or accumulated even to significantly lower levels after $P$. rapae feeding. Nevertheless, jin1-7 and jar11 were similarly attractive to $P$. rapae larvae in the two-choice 


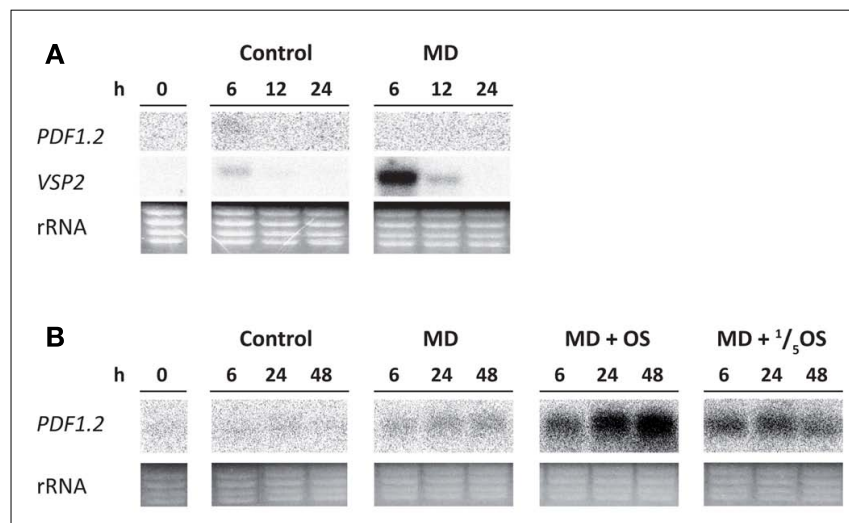

FIGURE 5 | Oral secretion of $\boldsymbol{P}$ rapae activates the ERF-branch of the JA pathway in mechanically damaged plants. Northern blot analysis of PDF1.2 and VSP2 transcript levels at different time points after mechanical damage (MD) (A) or MD in combination with treatment with water, or undiluted or fivefold diluted oral secretion (OS) of $P$. rapae at the sites of MD (B). Wounds were created by puncturing three small holes $(1 \mathrm{~mm}$ diameter) in each leaf using a sterile needle. Subsequently, $1 \mu \mathrm{L}$ of water or OS was applied to each wound. To check for equal loading, rRNA bands were stained with ethidium bromide. The experiments were repeated with similar results.

assays (Figure 4B). Together, these results suggest that the differences observed in glucosinolate content are not causally related to the feeding preference of the caterpillars in the two-choice assays.

JA-insensitive coil-1 plants had lower constitutive levels of aliphatic and indole glucosinolates than wild-type Col-0 plants, and also the P. rapae-induced levels were lower for a number of the glucosinolate compounds (Figure 6; Table 1). It can, thus, be suggested that the greater preference of P. rapae larvae for coil-1 compared to Col-0 (Figure 4) is at least partly due to the differences in glucosinolate content.

\section{DISCUSSION}

\section{MYC2 AND ORA59 SHAPE PLANT DEFENSES UPON HERBIVORY}

The JA signaling pathway differentially regulates plant defenses in response to insect herbivores and necrotrophic pathogens. Here, we investigated the molecular basis and biological function of the differential JA response as triggered in Arabidopsis by the specialist insect herbivore $P$. rapae. Feeding by $P$. rapae activated the expression of the MYC2-branch of the JA pathway (exemplified by the activation of the TF gene MYC2 and the marker gene VSP2), while the ERF-branch, which is typically activated in response to necrotrophic pathogens, was repressed (exemplified by the suppression of the ERF TF gene ORA59 and the marker gene PDF1.2). In MYC2-impaired jin1 plants, this herbivore-induced differential JA response was redirected toward the ERF-branch (Figure 1). A similar redirection toward the ERF-branch of the pathway was also observed in jar1-1 plants, which have the dual phenotype of reduced synthesis of JA-Ile and reduced MYC2-branch activation (Chini et al., 2007; Thines et al., 2007; Chung et al., 2008). Using knockdown RNAi-ORA59 and overexpression 35S:ORA59 lines, we provide evidence that the antagonism between the MYC2- and the ERF-branch during insect herbivory is regulated at the level of

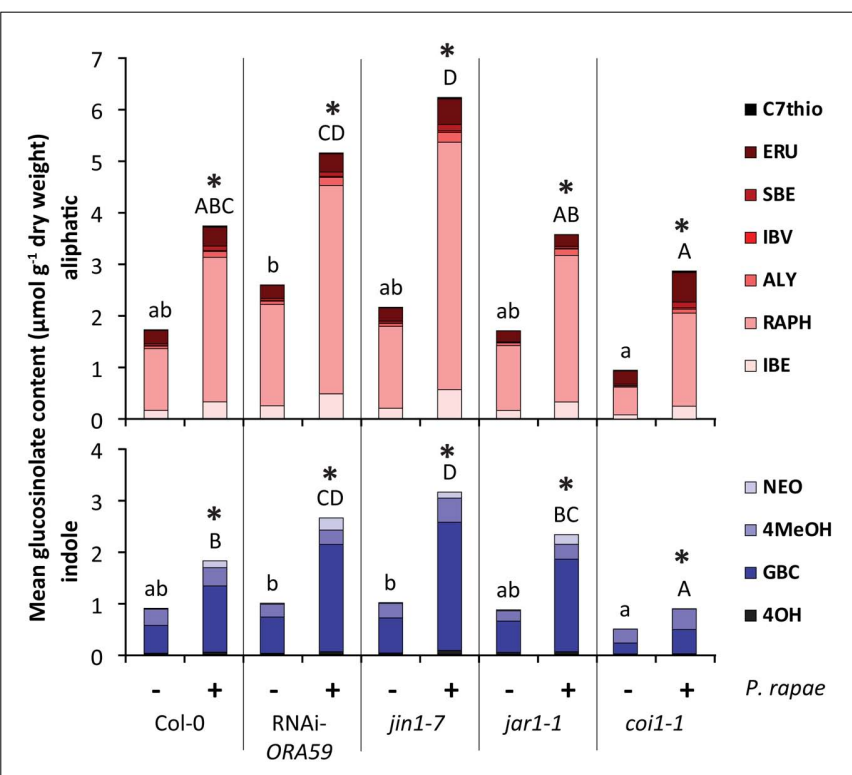

FIGURE 6 | Glucosinolate content in different Arabidopsis genotypes after $\mathbf{4}$ days of feeding by $\boldsymbol{P}$ rapae. Contents of aliphatic and indole glucosinolates of 6-week-old Col-0, coi1-1, jin 1-7, jar1-1, and RNAi-ORA59 plants after 4 days of feeding by first-instar larvae of $P$. rapae $(+)$. Controls $(-)$ show glucosinolate contents in non-infested plants. Asterisks indicate statistically significant differences between total constitutive and induced glucosinolate contents within a genotype (Student's $t$-test; $p<0.05$ ). Different letters indicate statistically significant differences in total glucosinolate contents between wild-type Col-0 and the JA signaling mutants and transgenics that received the same treatment (Tukey Post hoc test; $p<0.05$ ). C7thio, Methyl-thioheptyl; ERU, Glucoerucin; SBE,

Glucosiberin; IBV, Glucoiberverin; ALY, Glucoalyssin; RAPH, Glucoraphanin; IBE, Glucoiberin; NEO, Neo-glucobrassicin; 4MeOH, 4-Methoxy-

glucobrassicin; GBC, Glucobrassicin; 4OH, 4-Hydroxy-glucobrassicin.

MYC2 and ORA59 TF gene expression (Figure 2). Furthermore, our results provide detailed biological evidence that the previously described antagonism between MYC2 and ERF-type TFs (Lorenzo et al., 2003, 2004) is important for the differential regulation of the JA response as observed during plant-insect interactions. Using a two-choice assay, we show that $P$. rapae larvae preferred to feed on plants that express the ERF-branch, while expression of the MYC2branch had no effect on the preference of P. rapae (Figure 4). We thus conclude that by prioritizing the MYC2-branch of the JA pathway over the ERF-branch, Arabidopsis plants rewire the JA signaling pathway away from the $P$. rapae-preferred ERF-branch of the JA pathway, possibly to minimize attractiveness of the leaf tissue for insect feeding. We found no evidence for a role of glucosinolates in the preference of $P$. rapae for the ERF-branch of the JA pathway (Figure 6; Table 1). The induced responses leading to feeding preference by the insects thus still remain elusive. Interestingly, application of oral secretion of $P$. rapae into mechanically damaged leaf tissue induced the ERF-branch of the JA pathway (Figure 5), suggesting that elicitors in the oral secretion are potentially capable of steering the JA response toward expression of the P. rapae-preferred ERF-branch. During the interaction of $P$. rapae larvae with wild-type Col-0 plants, the MYC2/ERF balance shifts, however, toward the MYC2-branch, indicating that the arms race 
Table 1 | Fold-difference of glucosinolate levels in non-treated and $P$. rapae-induced JA signaling mutants and transgenics relative to wild-type Col-0 plants ${ }^{1}$.

\begin{tabular}{|c|c|c|c|c|c|c|c|c|c|c|c|c|}
\hline & & \multicolumn{7}{|c|}{ Aliphatic } & \multicolumn{4}{|c|}{ Indole } \\
\hline & & IBE & RAPH & ALY & IBV & SBE & ERU & C7thio & $40 \mathrm{H}$ & GBC & $4 \mathrm{MeOH}$ & NEO \\
\hline \multirow{3}{*}{$\begin{array}{l}0 \\
\mathbb{2} \\
\frac{2}{0} \\
0 \\
0 \\
1\end{array}$} & jar1-1 & 1.0 & 1.1 & 1.0 & 0.4 & 0.4 & 0.8 & 0.2 & 1.5 & 1.1 & 0.6 & 1.3 \\
\hline & RNAi-ORA59 & 1.5 & 1.6 & 1.4 & 1.1 & 1.1 & 1.0 & 0.8 & 0.9 & 1.3 & 0.8 & 1.4 \\
\hline & coil-1 & 0.5 & 0.5 & 0.5 & 0.7 & 0.8 & 1.0 & 1.3 & 0.4 & 0.4 & 0.9 & 0.2 \\
\hline \multirow{3}{*}{$\begin{array}{l}0 \\
\frac{\pi}{0} \\
\frac{2}{0} \\
0 \\
+ \\
+\end{array}$} & jar1-1 & 1.0 & 1.0 & 1.1 & 0.4 & 0.5 & 0.6 & 0.2 & 1.2 & 1.4 & 0.8 & 1.4 \\
\hline & RNAi-ORA59 & 1.5 & 1.4 & 1.4 & 1.0 & 1.0 & 1.0 & 0.7 & 1.2 & 1.6 & 0.8 & 1.8 \\
\hline & coi1-1 & 0.8 & 0.6 & 0.7 & 1.3 & 1.3 & 1.6 & 1.3 & 0.3 & 0.4 & 1.1 & 0.1 \\
\hline
\end{tabular}

${ }^{1}$ Green filled cells indicate statistically significant higher induced levels than in Col-0, whereas red filled cells indicate statistically significant lower induced levels of the indicated glucosinolate compound (Dunnett's pairwise multiple comparison t-test, $p<0.05$ ).

between plant and attacker during this interaction is decided in favor of the plant.

\section{P. RAPAE LARVAE PREFER TO FEED ON PLANTS THAT EXPRESS THE ERF-BRANCH OF THE JA PATHWAY}

It is generally accepted that the JA signaling pathway is important in plant defense against herbivorous insects (Howe and Jander, 2008). Growth and development of $P$. rapae larvae was indeed significantly enhanced on JA-insensitive Arabidopsis coil-1 plants (Figure 3; Reymond et al., 2004; Bodenhausen and Reymond, 2007; Van Oosten et al., 2008). However, in comparison to the generalist herbivores Spodoptera exigua (Beet armyworm) and Spodoptera littoralis (Egyptian cotton worm), the effect on the performance of $P$. rapae larvae was relatively small (Bodenhausen and Reymond, 2007; Van Oosten et al., 2008), probably because this specialist herbivore is well adapted to the defense responses that are triggered in the wild-type plants (Wittstock et al., 2004). Also in a two-choice assay, $P$. rapae larvae had a significant preference for the JA-insensitive coil-1 plants (Figure 4D), again highlighting the role of JA in defense against insect feeding. In wild tobacco (Nicotiana attenuata) plants, silencing of COI1 resulted in greater damage levels inflicted by the local herbivore community in a field experiment (Paschold et al., 2007). Laboratory choice assays confirmed that the COI1-silenced wild tobacco plants were preferred by herbivores (Paschold et al., 2007), which is similar to what we observed with Arabidopsis.

In mutant Arabidopsis coil-1 plants, the JA signaling pathway is completely blocked. This is in contrast to JA response mutants such as jin1 and jar1, which still display a strong but altered JA response in response to insect herbivory. JA-Ile production is compromised in jar1-1 (Staswick and Tiryaki, 2004), and was found to be responsible for activating COI1-mediated degradation of JAZ repressors proteins that suppress MYC2 action (Chini et al., 2007; Thines et al., 2007; Chung et al., 2008). Previously, it was demonstrated that jar1-1 plants do not show significant differences in JA-responsive gene expression in response to wounding, probably because the reduced, albeit not nil, level of bioactive JA-Ile in wounded jar1-1 leaves exceeds the level needed to saturate the
JA-Ile-induced transcriptional response (Koo et al., 2009). Indeed, the MYC2-marker gene VSP2 was not significantly affected in $P$. rapae-infested jar1-1 plants (Figure 1). However, in contrast to P. rapae-infested Col-0 plants, the expression of PDF1.2 was not suppressed in jar1-1, indicating that the jar1-1 mutation significantly affects the MYC2-mediated suppression of the ERF-branch during insect feeding. The observation that jin1 and jarl plants were significantly more preferred by $P$. rapae larvae than wildtype Col-0 plants, and were similarly preferred when given a choice between both mutants (Figure 4), suggests that the phenotype that the mutants share, namely strong activation of the ERF-branch upon insect feeding, is crucial for the preference of $P$. rapae. These molecular and ecological phenotypes of jin 1 and jar 1 plants are shared with ORA59-overexpressing 35S:ORA59 plants, which also express the ERF-branch of the JA pathway and are also significantly more preferred by $P$. rapae larvae (Figures 2 and 4). Activation of the MYC2-branch in Col-0 did not make the plant less attractive than jin1-1/RNAi-ORA59 plants that were unable to activate either the MYC2- or the ERF-branch (Figure 4C). We thus conclude that $P$. rapae larvae were stimulated to feed from plants that express the ERF-branch rather then that they were being deterred by plants that express the MYC2-branch of the JA pathway.

\section{EFFECT OF THE DIFFERENTIAL JA RESPONSE ON INSECT FEEDING}

One of the ways by which caterpillars can discriminate for food quality is by taste. As measured by electrophysiological activity, chemoreceptors in taste sensilase of the larvae of $P$. rapae respond to several secondary plant substances, such as naringenin and strychnine. Choice tests revealed that these "bitter" compounds can be classified as deterrents to $P$. rapae larvae (Zhou et al., 2009). Besides taste cells specialized in deterrents, also cells responding to stimulatory feeding compounds have been discovered. Caterpillar species respond differentially to specific sugars and amino acids as a way to determine plant quality. As a specialist on glucosinolate-containing plants, $P$. rapae contains two glucosinolate-sensitive taste cells that aid the larvae to discriminate between glucosinolate-containing hosts and glucosinolate-lacking 
non-hosts (Schoonhoven and Van Loon, 2002). It has been proposed that wound-induced responses are important in determining the pattern of feeding by insect herbivores, in which the insect moves away from a site that is heavily induced for defense (Edwards and Wratten, 1983). In line with this, larvae should settle longer on plants containing a less detrimental induced defense, making it possible to study effectiveness of defense in choice tests. Our results suggest that $P$. rapae caterpillars stay longer on Arabidopsis plants in which the ERF-branch of the JA pathway is activated, suggesting that the ERF-branch regulates the production of metabolites that are feeding stimulants for the P. rapae larvae.

Glucosinolates are secondary metabolites consisting out of a $\beta$-thioglucose moiety, a sulfonated oxime moiety, and a variable side chain which greatly determines level of toxicity (Hopkins et al., 2009). Indole glucosinolates are derived from tryptophan, whereas aliphatic glucosinolates are derived from methionine. Glucosinolates are implicated in stimulating oviposition of $P$. rapae butterflies (De Vos et al., 2008). We tested whether differences in glucosinolate composition between different genotypes might account for the observed preference of the P. rapae larvae for plants expressing the ERF-branch of the JA pathway. However, although amounts of several induced individual glucosinolates differed in the tested mutants from wild-type, no evidence was found for a role for glucosinolates in the enhanced preference of caterpillars for leaf tissue expressing the ERF-branch of the JA pathway.

\section{REWIRING OF THE JA SIGNALING PATHWAY DURING THE ARABIDOPSIS-P. RAPAE INTERACTION}

Application of oral secretion of $P$. rapae into mechanically inflicted wounds triggered the ERF-branch of the JA pathway (Figure 5). This suggests that $P$. rapae oral secretion contains elicitors that trigger the herbivore-preferred ERF-branch of the JA pathway in wounded leaf tissue, which may be a mechanism by which these specialist herbivores attempt to manipulate the JA response to their own benefit. Nevertheless, feeding by P. rapae larvae on wildtype Col-0 plants finally results in suppression of the ERF-branch because the MYC2-branch of the JA pathway becomes dominantly activated during the Arabidopsis-P. rapae interaction. Apparently, crosstalk between the MYC2- and ERF-branch leads to rewiring of JA signaling in favor of the MYC2-branch that strongly antagonizes the herbivore-preferred ERF-branch of the JA pathway. In pharmacological experiments, this antagonistic effect has previously been demonstrated to involve ABA signaling, which in combination with JA signaling results in the prioritization of the MYC2-branch over the ERF-branch (Anderson et al., 2004; Lorenzo et al., 2004). Herbivory by $P$. rapae has previously been shown to be associated with ABA-responsive gene expression (Bodenhausen and Reymond, 2007). Hence, it is tempting to speculate that activation of the ABA pathway during $P$. rapae feeding is responsible for redirecting the JA pathway toward the MYC2-branch and away from the herbivore-preferred ERF-branch. Natural variation of the MYC2/ERF balance exists between $A$. thaliana accessions (Ahmad et al., 2011), but to what extent this correlates with the level of resistance to insect herbivores or their feeding preference remains to be elucidated.

More examples of insect herbivores that manipulate the host's defense response have been described, often with a favorable outcome for the attacker. For instance, components in the oral secretion of the generalist herbivore Helicoverpa zea was shown to reduce the JA-dependent synthesis of nicotine, a toxin that is produced by tobacco plants to defend themselves against herbivory (Musser et al., 2002). In Arabidopsis, Zarate et al. (2007) showed that phloem feeding silverleaf whiteflies (Bemisia tabaci) switch on the SA defense pathway that antagonizes the JA defense pathway that hinders whitefly nymph development. A similar phenomenon was observed with caterpillars from S. exigua. Elicitors from salivary excretions of this herbivore suppressed effectual JAdependent defenses through the activation of the SA pathway (Weech et al., 2008; Diezel et al., 2009). In Lima bean, infestation with the whitefly B. tabaci and the spider mite Tetranychus urticae was shown to suppress indirect JA-dependent defenses that were induced by the spider mites, resulting in reduced attraction of carnivorous enemies of the spider mites (Zhang et al., 2009). Recently, elicitors from insect eggs were found to activate the SA pathway in Arabidopsis at the site of oviposition. As a result, JA-dependent defenses were suppressed, resulting in an advantage for the newly hatched offspring that fed from the undefended tissue (Bruessow et al., 2010). These examples pinpoint hormone-regulated signaling pathways as important targets for plant attackers to manipulate the host's defense response. In addition, in the present study, we show that the plant evolved to recognize the invader so that the hormone signaling route that favors the invader is suppressed.

\section{MATERIALS AND METHODS PLANT GROWTH CONDITIONS}

Seeds of A. thaliana wild-type Col-0, glabrous Col-5( $g l 1)$, mutants jin1-1(gl1), jin1-1, jin1-2, jin1-7 (SALK_040500), jin1-10 (SALK_017005; Lorenzo et al., 2004), and jar1-1 (Staswick et al., 1992), and transgenics RNAi-ORA59 and 35S:ORA59 (Pré et al., 2008) were sown on quartz sand and, after 2 weeks of growth, seedlings were transplanted into $60-\mathrm{ml}$ pots containing a sandpotting soil mixture $(5: 12 \mathrm{v} / \mathrm{v})$ that had been autoclaved twice for 20 min with a 24-h interval (Van der Ent et al., 2008). Mutant coi1-1 (gl1) (Feys et al., 1994) and coil-1 plants were selected by size on vermiculite supplied with $50 \mu \mathrm{M}$ MeJA, after which the genotype was confirmed as described (Xie et al., 1998). Non-glabrous jin 1-1 and coil-1 were produced by back-crossing jin 1-1(gl1) and coil-1(gl1) with Col-0. For genotyping of the jin1-1 and coil1 mutation the following primers were used: jin1-1-Fw: $5^{\prime}-\mathrm{AAG}$ CCA GCA AAC GGT AGA GA-3'; jin1-1-Rv 5'-ACG CGA GAA GAG CTG AAG AA-3'; Fw-coil-1 5' -GAA AGG ATT ACA GAT CTG CC-3'; Rv-coil-1 5'-CAT ATT GGC TCC TTC AGG AC-3'. Wild-type was distinguished from coil-1 by digesting the 530-bp PCR product with $X \mathrm{cmI}$, which leaves the mutant PCR product intact (Xie et al., 1998). Plants were cultivated in a growth chamber with an 8 -h day $\left(200 \mu \mathrm{E} \mathrm{m}^{-2} \mathrm{~s}^{-1}\right.$ at $\left.24^{\circ} \mathrm{C}\right)$ and 16 -h night $\left(20^{\circ} \mathrm{C}\right)$ cycle at $70 \%$ relative humidity for another 4 weeks. Plants were watered every other day and received half-strength Hoagland solution (Hoagland and Arnon, 1938) containing $10 \mu \mathrm{M}$ Sequestreen (CIBA-Geigy, Basel, Switzerland) once a week.

\section{PIERIS RAPAE ASSAYS}

Pieris rapae (small cabbage white butterfly) was reared on Brussels sprouts plants (Brassica oleracea gemmifera cv. Icarus) as described 
previously (Van Poecke et al., 2001). In all experiments, first-instar (L1) larvae were used. For gene expression analysis, three larvae were placed separately on fully expanded leaves of 6-week-old Arabidopsis plants using a fine paint-brush. In all cases, larvae were removed $24 \mathrm{~h}$ after the start of the experiment. Leaves of damaged and undamaged plants were harvested at different time points after the start of caterpillar infestation.

Pieris rapae growth was monitored during a 10-day period after transfer of single larvae to individual 6-week-old Arabidopsis plant genotype $(n=40-45)$ that were placed inside a Magenta GA-7 vessel (Sigma-Aldrich, Zwijndrecht, The Netherlands) with a modified lid that contained an insect-proof mesh (Van Oosten et al., 2008). The caterpillars were weighed to the nearest $0.1 \mathrm{mg}$ on a microbalance (Sartorius AC211P) at 4, 7, and 10 days after the start of the experiment.

For $P$. rapae two-choice assays, two L1 larvae were released per plant in an arena $(n=20)$ that consisted of two 6-week-old Arabidopsis plants of each tested genotype. The plants in the two-choice arena were in physical contact, which allowed the caterpillars to freely move through the arena. After 4 days the number of caterpillars present on each genotype was monitored. The frequency distribution of the caterpillars over the different genotypes was calculated and tested for statistical difference from a 50/50 choice proportion using Student's $t$-test.

\section{WOUNDING ASSAYS}

The effect of wounding was assessed by mechanically damaging the leaf tissue of 6-week-old plants. Three spots were punctured in each leaf using a sterile needle ( $1 \mathrm{~mm}$ diameter). Oral secretion was collected from L4/L5 larvae that were allowed to feed on uninduced Col-0 plants for $24 \mathrm{~h}$ as described (Mattiacci et al., 1995). In each of the wounds, a 1- $\mu \mathrm{L}$ droplet of freshly collected, undiluted or $5 \times$ diluted oral secretion was applied. A similar volume of tap water was applied as a control.

\section{RNA EXTRACTION AND NORTHERN BLOT ANALYSIS}

Total RNA was isolated as described (Van Wees et al., 1999). For northern blot analysis, 10 or $15 \mu \mathrm{g}$ of RNA were denatured using glyoxal and dimethyl sulfoxide (Sambrook et al., 1989), electrophoretically separated on a $1.5 \%$ agarose gel, and blotted onto Hybond- $\mathrm{N}^{+}$membrane (Amersham, 's-Hertogenbosch, The Netherlands) by capillary transfer. The electrophoresis and blotting buffer consisted of 10 and $25 \mathrm{mM}$ sodium phosphate $(\mathrm{pH}$ 7.0), respectively. To check for equal loading, rRNA bands were

\section{REFERENCES}

Adie, B., Chico, J. M., Rubio-Somoza, I., and Solano, R. (2007). Modulation of plant defenses by ethylene. J. Plant Growth Regul. 26, 160-177.

Ahmad, S., Van Hulten, M., Martin, J., Pieterse, C. M., Van Wees, S. C., and Ton, J. (2011). Genetic dissection of basal defence responsiveness in accessions of Arabidopsis thaliana. Plant Cell Environ. 34, 1191-2006.

Anderson, J. P., Badruzsaufari, E., Schenk, P. M., Manners, J. M., Desmond, O. J., Ehlert, C.,

stained with ethidium bromide. Northern blots were hybridized with gene-specific probes for PDF1.2 and VSP2 and ORA59 as described previously (Leon-Reyes et al., 2010). A probe for MYC2 was made by PCR amplification of cDNA of MeJA-treated plants and the gene-specific primers MYC2-Fw 5'-GGTCA CCGGT TTATG GAATG- $3^{\prime}$ and MYC2-Rv $5^{\prime}$-CGTAT CCGTC ACCTC CTCAT- $3^{\prime}$. After hybridization with $\alpha-\left[{ }^{32} \mathrm{P}\right]$-dCTP-labeled probes, blots were exposed for autoradiography and signals quantified using a BioRad Molecular Imager FX with Quantity One software (BioRad, Veenendaal, The Netherlands). The AGI numbers for the genes studied are At5g44420 (PDF1.2), At5g24770 (VSP2), Atlg06160 (ORA59), and At1g32640 (MYC2). All gene expression analyses have been repeated with similar results.

\section{RT-qPCR}

SuperScript ${ }^{\mathrm{TM}}$ III Reverse Transcriptase was used to convert DNAfree total RNA into cDNA. PCR reactions were performed in optical 96-well plates (Applied Biosystems) with an ABI PRISM ${ }^{\circledR}$ 7900 HT sequence detection system, using SYBR ${ }^{\circledR}$ Green to monitor the synthesis of double-stranded DNA. A standard thermal profile was used: $50^{\circ} \mathrm{C}$ for $2 \mathrm{~min}, 95^{\circ} \mathrm{C}$ for $10 \mathrm{~min}, 40$ cycles of $95^{\circ} \mathrm{C}$ for $15 \mathrm{~s}$, and $60^{\circ} \mathrm{C}$ for $1 \mathrm{~min}$. Amplicon dissociation curves were recorded after cycle 40 by heating from 60 to $95^{\circ} \mathrm{C}$ with a ramp speed of $1.9^{\circ} \mathrm{C} \mathrm{min}^{-1}$. ORA59 transcript levels (primer pair from Czechowski et al., 2004) were calculated relative to the reference gene Atlg13320 (Czechowski et al., 2005) using the $2^{-\Delta \Delta \mathrm{Ct}}$ method described previously (Livak and Schmittgen, 2001; Schmittgen and Livak, 2008).

\section{GLUCOSINOLATE MEASUREMENTS}

Caterpillar-damaged leaves (infested with two L1 larvae for 4 days) were harvested from 6-week-old plants, frozen in liquid nitrogen and subsequently freeze-dried. Plant material was homogenized and a 100-mg aliquot was weighed in a 2-ml tube for glucosinolate extraction, as described previously (Kabouw et al., 2010). For each treatment, five replicas of a pool of three plants were used.

\section{ACKNOWLEDGMENTS}

The authors thank Dennis Mourik for technical assistance and Leo Koopman, André Gidding, Leon Westerd, and Hans van Pelt for insect rearing. This research was supported by VICI grant 865.04.002 of the Earth and Life Sciences Foundation (ALW), which is subsidized by The Netherlands Organization of Scientific Research (NWO).

Bodenhausen, N., and Reymond, P. (2007). Signaling pathways controlling induced resistance to insect herbivores in Arabidopsis. Mol. Plant Microbe Interact. 20, 1406-1420.

Bruessow, F., Gouhier-Darimont, C., Buchala, A., Métraux, J.-P., and Reymond, P. (2010). Insect eggs suppress plant defence against chewing herbivores. Plant J. 62, 876-885.

Chini, A., Fonseca, S., Fernandez, G., Adie, B., Chico, J. M., Lorenzo, O., Garcia-Casado, G., Lopez-Vidriero, I., Lozano, F. M., Ponce, M. R., Micol,
J. L., and Solano, R. (2007). The JAZ family of repressors is the missing link in jasmonate signalling. Nature 448, 666-671.

Chung, H. S., Koo, A. J., Gao, X., Jayanty, S., Thines, B., Jones, A. D., and Howe, G. A. (2008). Regulation and function of Arabidopsis JASMONATE ZIM-domain genes in response to wounding and herbivory. Plant Physiol. 146, 952-964.

Czechowski, T., Bari, R. P., Stitt, M., Scheible, W. R., and Udvardi, M. K. (2004). Real-time RT-PCR profiling of over 1400 Arabidopsis 
transcription factors: unprecedented sensitivity reveals novel rootand shoot-specific genes. Plant J. 38, 366-379.

Czechowski, T., Stitt, M., Altmann, T., Udvardi, M. K., and Scheible, W.R. (2005). Genome-wide identification and testing of superior reference genes for transcript normalization in Arabidopsis. Plant Physiol. 139, 5-17.

De Vos, M., Kriksunov, K. L., and Jander, G. (2008). Indole-3-acetonitrile production from indole glucosinolates deters oviposition by Pieris rapae. Plant Physiol. 146, 916-926.

De Vos, M., Van Oosten, V. R., Van Poecke, R. M., Van Pelt, J. A., Pozo, M. J., Mueller, M. J., Buchala, A. J., Métraux, J. P., Van Loon, L. C., Dicke, M., and Pieterse, C. M. (2005). Signal signature and transcriptome changes of Arabidopsis during pathogen and insect attack. Mol. Plant Microbe Interact. 18, 923-937.

De Vos, M., Van Zaanen, W., Koornneef, A., Korzelius, J. P., Dicke, M., Van Loon, L. C., and Pieterse, C. M. (2006). Herbivore-induced resistance against microbial pathogens in Arabidopsis. Plant Physiol. 142, 352-363.

Dicke, M., Van Loon, J. J., and Soler, R. (2009). Chemical complexity of volatiles from plants induced by multiple attack. Nat. Chem. Biol. 5, 317-324.

Diezel, C., Von Dahl, C. C., Gaquerel, E., and Baldwin, I. T. (2009). Different lepidopteran elicitors account for cross-talk in herbivory-induced phytohormone signaling. Plant Physiol. 150, 1576-1586.

Dombrecht, B., Xue, G. P., Sprague, S. J., Kirkegaard, J. A., Ross, J. J., Reid, J. B., Fitt, G. P., Sewelam, N., Schenk, P. M., Manners, J. M., and Kazan, K. (2007). MYC2 differentially modulates diverse jasmonate-dependent functions in Arabidopsis. Plant Cell 19, 2225-2245

Edwards, P. J., and Wratten, S. D. (1983). Wound induced defenses in plants and their consequences for patterns of insect grazing. Oecologia 59, 88-93.

Ehrlich, P. R., and Raven, P. H. (1964). Butterflies and plants: a study in coevolution. Evolution 18, 586-608.

Fernandez-Calvo, P., Chini, A., Fernandez-Barbero, G., Chico, J. M., Gimenez-Ibanez, S., Geerinck, J., Eeckhout, D., Schweizer, F., Godoy, M., Franco-Zorrilla, J. M., Pauwels, L., Witters, E., Puga, M. I., Paz-Ares, J., Goossens, A., Reymond, P., De Jaeger, G., and Solano, R. (2010). The Arabidopsis bHLH transcription factors MYC3 and MYC4 are targets of JAZ repressors and act additively with MYC2 in the activation of jasmonate responses. Plant Cell 23, 701-715.

Feys, B., Benedetti, C. E., Penfold, C. N., and Turner, J. G. (1994). Arabidopsis mutants selected for resistance to the phytotoxin coronatine are male sterile, insensitive to methyl jasmonate, and resistant to a bacterial pathogen. Plant Cell 6, 751-759.

Fonseca, S., Chini, A., Hamberg, M., Adie, B., Porzel, A., Kramell, R., Miersch, O., Wasternack, C., and Solano, R. (2009). (+)-7iso-Jasmonoyl-L-isoleucine is the endogenous bioactive jasmonate. Nat. Chem. Biol. 5, 344-350.

Gfeller, A., Dubugnon, L., Liechti, R., and Farmer, E. E. (2010). Jasmonate biochemical pathway. Sci. Signal. 3, $\mathrm{cm} 3$.

Glazebrook, J. (2005). Contrasting mechanisms of defense against biotrophic and necrotrophic pathogens. Annu. Rev. Phytopathol. 43, 205-227.

Hoagland, D. R., and Arnon, D. I. (1938). The water culture method for growing plants without soil. Calif. Agr. Exp. Sta. Circ. 347, 36-39.

Hopkins, R. J., Van Dam, N. M., and Van Loon, J. J. (2009). Role of glucosinolates in insect-plant relationships and multitrophic interactions. Annu. Rev. Entomol. 54, 57-83.

Howe, G. A., and Jander, G. (2008). Plant immunity to insect herbivores. Annu. Rev. Plant Biol. 59, 41-66.

Jones, J. D., and Dangl, J. L. (2006). The plant immune system. Nature 444, 323-329.

Kabouw, P., Biere, A., Van der Putten, W. H., and Van Dam, N. M. (2010). Intra-specific differences in root and shoot glucosinolate profiles among white cabbage (Brassica oleracea var. capitata) cultivars. J. Agric. Food Chem. 58, 411-417.

Kant, M. R., Sabelis, M. W., Haring, M. A., and Schuurink, R. C. (2008). Intraspecific variation in a generalist herbivore accounts for differential induction and impact of host plant defences. Proc. Biol. Sci. 275, 443-452.

Kazan, K., and Manners, J. M. (2008). Jasmonate signaling: toward an integrated view. Plant Physiol. 146, 1459-1468.

Koo, A. J., Gao, X., Jones, A. D., and Howe, G. A. (2009). A rapid wound signal activates the systemic synthesis of bioactive jasmonates in Arabidopsis. Plant J. 59, 974-986.

Koo, A. J., and Howe, G. A. (2009). The wound hormone jasmonate. Phytochemistry 70, 1571-1580.
Koornneef, A., Leon-Reyes, A., Ritsema, T., Verhage, A., Den Otter, F. C., Van Loon, L. C., and Pieterse, C. M. (2008). Kinetics of salicylatemediated suppression of jasmonate signaling reveal a role for redox modulation. Plant Physiol. 147, 1358-1368.

Laluk, K., and Mengiste, T. (2010). Necrotroph attacks on plants: wanton destruction or covert extortion? Arabidopsis Book 8, e0136.

Leon-Reyes, A., Du, Y., Koornneef, A., Proietti, S., Körbes, A. P. Memelink, J., Pieterse, C. M., and Ritsema, T. (2010). Ethylene signaling renders the jasmonate response of Arabidopsis insensitive to future suppression by salicylic acid. Mol. Plant Microbe Interact. 23 187-197.

Leon-Reyes, A., Spoel, S. H., De Lange, E. S., Abe, H., Kobayashi, M., Tsuda, S., Millenaar, F. F., Welschen, R. A., Ritsema, T., and Pieterse, C. M. (2009). Ethylene modulates the role of NONEXPRESSOR OF PATHOGENESIS-RELATED GENES1 in cross talk between salicylate and jasmonate signaling. Plant Physiol. 149, 1797-1809.

Livak, K. J., and Schmittgen, T. D. (2001). Analysis of relative gene expression data using realtime quantitative PCR and the $2^{-\Delta \Delta C T}$ method. Methods 25, 402-408.

Lorenzo, O., Chico, J. M., SanchezSerrano, J. J., and Solano, R. (2004). JASMONATE-INSENSITIVE1 encodes a MYC transcription factor essential to discriminate between different jasmonate-regulated defense responses in Arabidopsis. Plant Cell 16, 1938-1950.

Lorenzo, O., Piqueras, R., SánchezSerrano, J. J., and Solano, R. (2003). ETHYLENE RESPONSE FACTOR1 integrates signals from ethylene and jasmonate pathways in plant defense. Plant Cell 15, 165-178.

Lorenzo, O., and Solano, R. (2005). Molecular players regulating the jasmonate signalling network. Curr. Opin. Plant Biol. 8, 532-540.

Mattiacci, L., Dicke, M., and Posthumus, M. A. (1995). ß-Glucosidase: an elicitor of herbivore-induced plant odor that attracts host-searching parasitic wasps. Proc. Natl. Acad. Sci. U.S.A. 92, 2036-2040.

Memelink, J. (2009). Regulation of gene expression by jasmonate hormones. Phytochemistry 70, 1560-1570.

Moreno, J. E., Tao, Y., Chory, J., and Ballare, C. L. (2009). Ecological modulation of plant defense via phytochrome control of jasmonate sensitivity. Proc. Natl. Acad. Sci. U.S.A. 106, 4935-4940.

Musser, R. O., Hum-Musser, S. M., Eichenseer, H., Peiffer, M., Ervin, G., Murphy, J. B., and Felton, G. W. (2002). Herbivory: caterpillar saliva beats plant defences - a new weapon emerges in the evolutionary arms race between plants and herbivores. Nature 416, 599-600.

Paschold, A., Halitschke, R., and Baldwin, I. T. (2007). Co(i) ordinating defenses: NaCOI1 mediates herbivore-induced resistance in Nicotiana attenuata and reveals the role of herbivore movement in avoiding defenses. Plant J. 51, 79-91.

Pauwels, L., Inzé, D., and Goossens, A. (2009). Jasmonate-inducible gene: what does it mean? Trends Plant Sci. 14, 87-91.

Penninckx, I. A., Thomma, B. P., Buchala, A., Métraux, J.-P., and Broekaert, W. F. (1998). Concomitant activation of jasmonate and ethylene response pathways is required for induction of a plant defensin gene in Arabidopsis. Plant Cell 10, 2103-2113.

Pieterse, C. M., and Dicke, M. (2007). Plant interactions with microbes and insects: from molecular mechanisms to ecology. Trends Plant Sci. 12, 564-569.

Pieterse, C. M., Leon-Reyes, A., Van Der Ent, S., and Van Wees, S. C. M. (2009). Networking by small-molecule hormones in plant immunity. Nat. Chem. Biol. 5, 308-316.

Pozo, M. J., Van der Ent, S., Van Loon, L. C., and Pieterse, C. M. (2008). Transcription factor MYC2 is involved in priming for enhanced defense during rhizobacteria-induced systemic resistance in Arabidopsis thaliana. New Phytol. 180, 511-523.

Pré, M., Atallah, M., Champion, A., De Vos, M., Pieterse, C. M., and Memelink, J. (2008). The AP2/ERF domain transcription factor ORA59 integrates jasmonic acid and ethylene signals in plant defense. Plant Physiol. 147, 1347-1357.

Reymond, P., Bodenhausen, N., Van Poecke, R. M., Krishnamurthy, V., Dicke, M., and Farmer, E. E. (2004). A conserved transcriptional pattern in response to a specialist and a generalist herbivore. Plant Cell 16, 3132-3147.

Reymond, P., Weber, H., Damond, M., and Farmer, E. E. (2000). Differential gene expression in response to mechanical wounding and insect feeding in Arabidopsis. Plant Cell 12 , 707-719. 
Sambrook, J., Fritsch, E. F., and Maniatis, T. (1989). Molecular Cloning: A Laboratory Manual, 2nd Edn. Cold Spring Harbor, NY: Cold Spring Harbor Laboratory Press.

Schmittgen, T. D., and Livak, K. J. (2008). Analyzing real-time PCR data by the comparative C-T method. Nat. Protoc. 3, 1101-1108.

Schoonhoven, L. M., and Van Loon, J. J. (2002). An inventory of taste in caterpillars: each species its own key. Acta Zool. Academ. Sci. Hung. 48, 215-263.

Schoonhoven, L. M., Van Loon, J. J., and Dicke, M. (2005). Insect-Plant Biology. Oxford: Oxford University Press.

Sheard, L. B., Tan, X., Mao, H. B., Withers, J., Ben-Nissan, G., Hinds, T. R., Kobayashi, Y., Hsu, F.-F., Sharon, M., Browse, J., He, S. Y., Rizo, J., Howe, G. A., and Zheng, N. (2010). Jasmonate perception by inositol-phosphatepotentiated COI1-JAZ co-receptor. Nature 468, 400-405.

Staswick, P. E., and Tiryaki, I. (2004). The oxylipin signal jasmonic acid is activated by an enzyme that conjugates it to isoleucine in Arabidopsis. Plant Cell 16, 2117-2127.

Staswick, P. E., Yuen, G. Y., and Lehman, C. C. (1992). Methyl jasmonate inhibition of root growth and induction of a leaf protein are decreased in an Arabidopsis thaliana mutant. Proc. Natl. Acad. Sci. U.S.A. 89, 6837-6840.

Thines, B., Katsir, L., Melotto, M., Niu, Y., Mandaokar, A., Liu, G. H., Nomura, K., He, S. Y., Howe, G. A., and Browse, J. (2007). JAZ repressor proteins are targets of the SCFCOI1 complex during jasmonate signalling. Nature 448, 661-665.

Thompson, J. N. (1994). The Coevolutionary Process. Chicago: The University of Chicago Press.
Van der Ent, S., Van Hulten, M., Pozo, M. J., Czechowski, T., Udvardi, M. K., Pieterse, C. M., and Ton, J. (2009). Priming of plant innate immunity by rhizobacteria and $B$ aminobutyric acid: differences and similarities in regulation. New Phytol. 183, 419-431.

Van der Ent, S., Verhagen, B. W., Van Doorn, R., Bakker, D., Verlaan, M. G., Pel, M. J., Joosten, R. G., Proveniers, M. C., Van Loon, L. C., Ton, J., and Pieterse, C. M. (2008). MYB72 is required in early signaling steps of rhizobacteria-induced systemic resistance in Arabidopsis. Plant Physiol. 146, 1293-1304.

Van Oosten, V. R., Bodenhausen, N., Reymond, P., Van Pelt, J. A., Van Loon, L. C., Dicke, M., and Pieterse, C. M. (2008). Differential effectiveness of microbially induced resistance against herbivorous insects in Arabidopsis. Mol. Plant Microbe Interact. 21, 919-930.

Van Poecke, R. M., Posthumus, M. A., and Dicke, M. (2001). Herbivoreinduced volatile production by Arabidopsis thaliana leads to attraction of the parasitoid Cotesia rubecula: chemical, behavioral, and geneexpression analysis. J. Chem. Ecol.27, 1911-1928.

Van Wees, S. C., Luijendijk, M., Smoorenburg, I., Van Loon, L. C., and Pieterse, C. M. (1999). Rhizobacteria-mediated induced systemic resistance (ISR) in Arabidopsis is not associated with a direct effect on expression of known defense-related genes but stimulates the expression of the jasmonate-inducible gene Atvsp upon challenge. Plant Mol. Biol. 41, 537-549.

Verhage, A., Van Wees, S. C., and Pieterse, C. M. (2010). Plant immunity: it's the hormones talking, but what do they say? Plant Physiol. 154, 536-540.

Walling, L. L. (2008). Avoiding effective defenses: strategies employed by phloem-feeding insects. Plant Physiol. 146, 859-866.

Wasternack, C. (2007). Jasmonates: an update on biosynthesis, signal transduction and action in plant stress response, growth and development. Ann. Bot. 100, 681-697.

Weech, M. H., Chapleau, M., Pan, L., Ide, C., and Bede, J. C. (2008). Caterpillar saliva interferes with induced Arabidopsis thaliana defence responses via the systemic acquired resistance pathway. J. Exp. Bot. 59, 2437-2448.

Wittstock, U., Agerbirk, N., Stauber, E. J., Olsen, C. E., Hippler, M., Mitchell-Olds, T., Gershenson, J., and Vogel, H. (2004). Successful herbivore attack due to metabolic diversion of a plant chemical defense. Proc. Natl. Acad. Sci. U.S.A. 101, 4859-4864.

Xie, D. X., Feys, B. F., James, S., Nieto-Rostro, M., and Turner, J. G. (1998). COI1: an Arabidopsis gene required for jasmonate-regulated defense and fertility. Science 280 , 1091-1094.

Yan, J., Zhang, C., Gu, M., Bai, Z., Zhang, W., Qi, T., Cheng, Z., Peng, W., Luo, H., Nan, F., Wang, Z., and Xie, D. (2009). The Arabidopsis CORONATINE INSENSITIVE1 protein is a jasmonate receptor. Plant Cell 21, 2220-2236.

Yano, S., and Ohsaki, N. (1993). The phenology and intrinsic quality of wild crucifers that determine the community structure of their herbivorous insects. Res. Popul. Ecol. (Kyoto) 35, 151-170.

Zarate, S. I., Kempema, L. A., and Walling, L. L. (2007). Silverleaf whitefly induces salicylic acid defenses and suppresses effectual jasmonic acid defenses. Plant Physiol. 143, 866-875.

Zhang, P. J., Zheng, S. J., Van Loon, J. J., Boland, W., David, A., Mumm, R., and Dicke, M. (2009). Whiteflies interfere with indirect plant defense against spider mites in Lima bean. Proc. Natl. Acad. Sci. U.S.A. 106, 21202-21207.

Zhou, D.-S., Wang, C. Z., and Van Loon, J. J. (2009). Chemosensory basis of behavioural plasticity in response to deterrent plant chemicals in the larva of the small cabbage white butterfly Pieris rapae. J. Insect Physiol. 55, 788-792.

Conflict of Interest Statement: The authors declare that the research was conducted in the absence of any commercial or financial relationships that could be construed as a potential conflict of interest.

Received: 15 June 2011; accepted: 19 August 2011; published online: 26 September 2011.

Citation: Verhage A, Vlaardingerbroek I, Raaymakers C, Van Dam NM, Dicke $M$, Van Wees SCM and Pieterse CMJ (2011) Rewiring of the jasmonate signaling pathway in Arabidopsis during insect herbivory. Front. Plant Sci. 2:47. doi: 10.3389/fpls.2011.00047

This article was submitted to Frontiers in Plant-Microbe Interaction, a specialty of Frontiers in Plant Science.

Copyright $\odot 2011$ Verhage, Vlaardingerbroek, Raaymakers, Van Dam, Dicke, Van Wees and Pieterse. This is an openaccess article subject to a non-exclusive license between the authors and Frontiers Media SA, which permits use, distribution and reproduction in other forums, provided the original authors and source are credited and other Frontiers conditions are complied with. 